\title{
A remark on the Dunkl differential-difference operators
}

by G.J. Heckman, University of Nijmegen.

\section{$\S 1$. Introduction}

Let $E$ be a Euclidean vector space of dimension $n$ with inner product $(\cdot, \cdot)$. For $\alpha \in E$ with $(\alpha, \alpha)=2$ we write

$$
r_{\alpha}(\lambda)=\lambda-(\alpha, \lambda) \alpha, \lambda \in E
$$

for the orthogonal reflection in the hyperplane perpendicular to $\alpha$.

Definition 1.1. A normalized root system $R$ in $E$ is a finite set of non zero vectors in $E$, normalized by $(\alpha, \alpha)=2 \quad \forall \alpha \in R$, such that $r_{\alpha}(\beta) \in R \quad \forall \alpha, \beta \in R$.

Let $R \subset E$ be a normalized root system. We write $W=W(R)$ for the group generated by the reflections $r_{\alpha}, \alpha \in R$. Denote by $\mathbb{C}[E]$ the algebra of $\mathbb{C}$-valued polynomial functions on $E$. For $w \in W, \xi \in E, \alpha \in R$ introduce the operators

$$
w, \partial_{\xi}, \Delta_{\alpha}: \mathbb{C}[E] \longrightarrow \mathbb{C}[E]
$$

by

$$
\begin{gathered}
(w p)(\lambda)=p\left(w^{-1} \lambda\right) \\
\left(\partial_{\xi} p\right)(\lambda)=\frac{d}{d t}\{p(\lambda+t \xi)\}_{t=0} \\
\left(\Delta_{\alpha} p\right)(\lambda)=\frac{p(\lambda)-p\left(r_{\alpha} \lambda\right)}{(\alpha, \lambda)} .
\end{gathered}
$$

Remark 1.2. The operators $\Delta_{\alpha}, \alpha \in R$ were studied by Bernstein, Gel'fand and Gel'fand and are related to the Schubert cells and the cohomology of G/P [BGG]. They are the infinitesimal analogues of the Demazure operators [De 1,2].

Let $R_{+}=\{\alpha \in R ;(\alpha, \lambda)>0\}$ for some fixed generic $\lambda \in E$ be a positive subsystem of $R$. 
Definition 1.3. Suppose for $\alpha \in R$ we have given $k_{\alpha} \in \mathbb{C}$ with $k_{w \alpha}=k_{\alpha} \forall w \in$ $W, \forall \alpha \in R$. For $\xi \in E$ the operator

$$
D_{\xi}=\partial_{\xi}+\sum_{\alpha \in R_{+}} k_{\alpha}(\alpha, \xi) \Delta_{\alpha}: \mathbb{C}[E] \longrightarrow \mathbb{C}[E]
$$

is called the Dunkl differential-difference operator.

Remark 1.4. It is easy to see that $D_{\xi}$ is independent of the choice of the positive subsystem $R_{+} \subset R$. If we write $q_{\alpha}=e^{2 \pi i k_{\alpha}}$ then one can think of the operator $D_{\xi}$ as a $q$-analogue (corresponding to the case $k_{\alpha} \rightarrow 0$ ) of the directional derivative $\partial_{\xi}$. We also write $D_{\xi}=D_{\xi}(k)$ to indicate the dependence on $k \in K=\left\{k=\left(k_{\alpha}\right)_{\alpha \in R} \in\right.$ $\left.\mathbb{C}^{R} ; k_{w \alpha}=k_{\alpha} \forall w \in W, \forall \alpha \in R\right\}$.

Theorem 1.5 (Dunkl [Du]): We have $D_{\xi} D_{\eta}=D_{\eta} D_{\xi} \forall \xi, \eta \in E$.

Let $\mathbb{C}\left[E^{*}\right]$ be the symmetric algebra on $E$. For $\pi \in \mathbb{C}\left[E^{*}\right]$ we write $\partial_{\pi}$ when we think of $\pi$ as a constant coefficient differential operator on $E$ (rather than a polynomial function on $E^{*}$ ). In view of Theorem 1.5 the constant coefficient differential operator $\partial_{\pi}$ has a well defined $q$-analogue

$$
D_{\pi}: \mathbb{C}[E] \longrightarrow \mathbb{C}[E]
$$

defined for a monomial $\pi=\xi_{1}^{d_{1}} \ldots \xi_{n}^{d_{n}}$ by

$$
D_{\pi}=D_{\pi}(k)=D_{\xi_{1}}^{d_{1}} \ldots D_{\xi_{n}}^{d_{n}}
$$

and extended by linearity.

Theorem 1.6 (Dunkl [Du]): Suppose $\xi_{1}, \ldots, \xi_{n}$ is an orthonormal basis for $E$. The $q$-analogue of the Laplacian is given by

$$
\sum_{j=1}^{n} D_{\xi_{j}}^{2}=\sum_{j=1}^{n} \partial_{\xi_{j}}^{2}+2 \sum_{\alpha \in R_{+}} k_{\alpha} \frac{1}{(\alpha, \cdot)}\left\{\partial_{\alpha}-\Delta_{\alpha}\right\} .
$$

In Section 2 we review the proofs of both theorems as given by Dunkl.

We write $\mathbb{C}[E]^{W}$ and $\mathbb{C}\left[E^{*}\right]^{W}$ for the space of $W$-invariants in $\mathbb{C}[E]$ and $\mathbb{C}\left[E^{*}\right]$ respectively. We denote by $\mathbb{A}$ the associative algebra of endomorphisms of $\mathbb{C}[E]$ generated by (multiplication by) $(\xi, \cdot)$ and $D_{\eta}$ for $\xi, \eta \in E$. Let $\mathbb{A}^{W}=\{D \in \mathbb{A} ; w D=D w \forall w \in W\}$ be the subalgebra of $W$-invariant operators in $\mathbb{A}$, and denote by

$$
\operatorname{Res}(D): \mathbb{C}[E]^{W} \longrightarrow \mathbb{C}[E]^{W}, D \in \mathbb{A}^{W}
$$


the restriction of $D$ to $\mathbb{C}[E]^{W}$. Clearly Res : $\mathbb{A}^{W} \rightarrow \operatorname{End}\left(\mathbb{C}[E]^{W}\right)$ is a homomorphism of algebras. Since $w D_{\xi} w^{-1}=D_{w \xi} \forall w \in W, \forall \xi \in E$ we have $D_{\pi} \in \mathbb{A}^{W} \forall \pi \in \mathbb{C}\left[E^{*}\right]^{W}$.

Theorem 1.7. Suppose by the Chevalley theorem that $\mathbb{C}[E]^{W}=\mathbb{C}\left[p_{1}, \ldots, p_{n}\right]$ with $p_{1}, \ldots, p_{n}$ homogeneous of degrees $d_{1} \leq \ldots \leq d_{n}$. Then the set

$$
\left\{\operatorname{Res}\left(D_{\pi}\right) ; \pi \in \mathbb{C}\left[E^{*}\right]^{W}\right\}
$$

is a commuting family of differential operators in the Weyl algebra $\mathbb{C}\left[k, p_{1}, \ldots, p_{n}\right.$, $\left.\frac{\partial}{\partial p_{1}}, \ldots, \frac{\partial}{\partial p_{n}}\right]$ containing the operator

$$
\operatorname{Res}\left(\sum_{j=1}^{n} D_{\xi_{j}}^{2}\right)=\sum_{j=1}^{n} \partial_{\xi_{j}}^{2}+2 \sum_{\alpha \in R_{+}} k_{\alpha} \frac{1}{(\alpha, \cdot)} \partial_{\alpha}
$$

Remark 1.8. The proof of this theorem is a triviality. However it can be reformulated as the complete integrability for the generalized non periodic Calogero-Moser system (both on the quantum mechanical level of differential operators and on the classical mechanical level of symbols). For root systems $R$ of type $A$ the complete integrability of the Calogero-Moser system was first established by Moser by realizing the system as a Lax pair [Mo]. The method of Moser was extended by Olshanetsky and Perelomov to cover the root systems $R$ of classical type [OP]. In the crystallographic case $(\alpha, \beta)^{2} \in \mathbb{Z}$ $\forall \alpha, \beta \in R$ the above theorem has been obtained before by Opdam using transcendental methods [HO, He1, Op 1,2, He 2].

Suppose $S \subset R$ is a set of roots in $R$ invariant under $W$. Let $S_{+}=S \cap R_{+}$and put

$$
\begin{aligned}
p_{S}(\cdot) & =\prod_{\alpha \in S_{+}}(\alpha, \cdot) \in \mathbb{C}[E] \\
\pi_{S} & =\prod_{\alpha \in S_{+}} \alpha \in \mathbb{C}\left[E^{*}\right] .
\end{aligned}
$$

Clearly we have

$$
w p_{S}=\chi(w) p_{S}, w \pi_{S}=\chi(w) \pi_{S} \quad \forall w \in W
$$

for some one dimensional character $\chi=\chi_{S}$ of $W$, and conversely every $p \in \mathbb{C}[E]$ with $w p=\chi(w) p \forall w \in W$ is divisible in $\mathbb{C}[E]$ by $p_{S}$. Although $p_{S}^{-1} D_{\pi_{S}}(k)$ need not be an endomorphism of $\mathbb{C}[E]$ it follows that $p_{S}^{-1} D_{\pi_{S}}(k)(p) \in \mathbb{C}[E]^{W} \forall p \in \mathbb{C}[E]^{W}$, and hence

$$
G\left(1_{S}, k\right):=\operatorname{Res}\left(p_{S}^{-1} D_{\pi_{S}}(k)\right) \in \operatorname{End}\left(\mathbb{C}[E]^{W}\right)
$$


is a well defined endomorphism of $\mathbb{C}[E]^{W}$. We also write

$$
G\left(-1_{S}, k\right):=\operatorname{Res}\left(D_{\pi_{S}}\left(k-1_{S}\right) \cdot p_{S}\right) \in \operatorname{End}\left(\mathbb{C}[E]^{W}\right)
$$

where $k-1_{S} \in K$ is the multiplicity function by $\left(k-1_{S}\right)_{\alpha}=k_{\alpha}-1$ for $\alpha \in S$ and $\left(k-1_{S}\right)_{\alpha}=k_{\alpha}$ for $\alpha \in R \backslash S$.

Theorem 1.9. The operators (1.16) and (1.17) are differential operators in the Weyl algebra $\mathbb{C}\left[k, p_{1}, \ldots, p_{n}, \frac{\partial}{\partial p_{1}}, \ldots, \frac{\partial}{\partial p_{n}}\right]$ and satisfy the shift relations

$$
\begin{gathered}
G\left(1_{S}, k\right) \operatorname{Res}\left(D_{\pi}(k)\right)=\operatorname{Res}\left(D_{\pi}\left(k+1_{S}\right)\right) G\left(1_{S}, k\right) \\
G\left(-1_{S}, k\right) \operatorname{Res}\left(D_{\pi}(k)\right)=\operatorname{Res}\left(D_{\pi}\left(k-1_{S}\right)\right) G\left(-1_{S}, k\right)
\end{gathered}
$$

$\forall \pi \in \mathbb{C}\left[E^{*}\right]^{W}$. Here $\left(k \pm 1_{S}\right)_{\alpha}=k_{\alpha} \pm 1 \forall \alpha \in S$ and $\left(k \pm 1_{S}\right)_{\alpha}=k_{\alpha} \forall \alpha \in R \backslash S$.

The proofs of both Theorem 1.7 and 1.9 will be given in Section 3 .

Remark 1.10. In the terminology of Opdam the operator (1.16) is a raising operator and the operator (1.17) a lowering operator for the commuting family (1.11). Again in the crystallographic case the above theorem was obtained by Opdam [Op 2]. Recall Macdonald's (infinitesimal) constant term conjecture, which says that for $\mathcal{R}(s)>0$

$$
\int_{E} \prod_{\alpha \in R_{+}}|(\alpha, \lambda)|^{2 s} d \gamma(\lambda)=\prod_{j=1}^{n} \frac{\left(s d_{j}\right) !}{s !}
$$

where $d \gamma(\lambda)=(2 \pi)^{-\frac{n}{2}} e^{-\frac{1}{2}(\lambda, \lambda)} d \lambda$ is the Gaussian measure on $E$ [Ma]. The same arguments as given in [Op 3, Section 6] show that the evaluation of this integral is equivalent with

$$
G(-1, k)(1)=|W| \cdot \prod_{i=1}^{n} \prod_{j=1}^{m_{i}}\left(d_{i} k-j\right)
$$

where $-1=-1_{R}$ and $k=k_{\alpha} \forall \alpha \in R$. In turn this latter formula is related to the normalization of the "multivariable Bessel function associated with $R$ " at $\xi=0$. This normalization problem has been analyzed by Opdam, and the desired formula (1.21) can be obtained [Op 4]. After this one can proceed as in [Op 3, Section 7] to compute the Bernstein-Sato polynomial of the discriminant without the crystallographic restriction in accordance with a conjecture of Yano and Sekiguchi [YS]. 


\section{$\S 2$. The Dunkl differential-difference operators.}

Using the bracket $[\cdot, \cdot]$ for the commutator of endomorphisms of $\mathbb{C}[E]$ we can write for $\xi, \eta \in E$

$$
\left[D_{\xi}, D_{\eta}\right]=I+I I+I I I
$$

with

$$
\begin{aligned}
I & =\left[\partial_{\xi}, \partial_{\eta}\right]=0 \\
I I & =\sum_{\alpha \in R_{+}} k_{\alpha}\left\{(\alpha, \xi)\left[\Delta_{\alpha}, \partial_{\eta}\right]+(\alpha, \eta)\left[\partial_{\xi}, \Delta_{\alpha}\right]\right\} \\
I I I & =\sum_{\alpha, \beta \in R_{+}} k_{\alpha} k_{\beta}(\alpha, \xi)(\beta, \eta)\left[\Delta_{\alpha}, \Delta_{\beta}\right] .
\end{aligned}
$$

Lemma 2.1. For $\xi \in E, \alpha \in R$ we have

$$
\left[\partial_{\xi}, \Delta_{\alpha}\right]=\frac{(\alpha, \xi)}{(\alpha, \cdot)}\left\{r_{\alpha} \partial_{\alpha}-\Delta_{\alpha}\right\}
$$

Proof: Using the definition $\Delta_{\alpha}=\frac{1}{(\alpha, \cdot)}\left(1-r_{\alpha}\right)$ we get

$$
\begin{aligned}
{\left[\partial_{\xi}, \Delta_{\alpha}\right] } & =\left[\partial_{\xi}, \frac{1}{(\alpha, \cdot)}\right]\left(1-r_{\alpha}\right)+\frac{1}{(\alpha, \cdot)}\left[\partial_{\xi}, 1-r_{\alpha}\right] \\
& =-\frac{(\alpha, \xi)}{(\alpha, \cdot)^{2}}\left(1-r_{\alpha}\right)+\frac{1}{(\alpha, \cdot)} r_{\alpha}\left(\partial_{\xi}-\partial_{r_{\alpha} \xi}\right) \\
& =-\frac{(\alpha, \xi)}{(\alpha, \cdot)} \Delta_{\alpha}+\frac{(\alpha, \xi)}{(\alpha, \cdot)} r_{\alpha} \partial_{\alpha} .
\end{aligned}
$$

Using (2.5) the second term (2.3) can be rewritten as

$$
I I=\sum_{\alpha \in R_{+}} k_{\alpha} \frac{(\alpha, \xi)(\alpha, \eta)}{(\alpha, \cdot)}\left\{r_{\alpha} \partial_{\alpha}-\Delta_{\alpha}\right\}(-1+1)=0 .
$$

The third term (2.4) can be written as

$$
I I I=\sum_{\alpha, \beta \in R_{+}} k_{\alpha} k_{\beta}\{(\alpha, \xi)(\beta, \eta)-(\alpha, \eta)(\beta, \xi)\} \Delta_{\alpha} \Delta_{\beta}
$$

and for the proof of Theorem 1.5 it remains to verify the vanishing of this third term.

Proposition 2.2. Suppose $B(\cdot, \cdot)$ is a bilinear form on $E$ such that

$$
B\left(r_{\alpha} \lambda, r_{\alpha} \mu\right)=B(\mu, \lambda) \quad \forall \lambda, \mu \in E, \forall \alpha \in R \cap \operatorname{span}\langle\lambda, \mu\rangle .
$$


If $w \in W$ is a pure rotation (i.e. $\operatorname{dim} \operatorname{Im}(w-\operatorname{Id})=2$ ) then

$$
\sum_{\alpha, \beta \in R_{+}, r_{\alpha} r_{\beta}=w} k_{\alpha} k_{\beta} B(\alpha, \beta) \frac{1}{(\alpha, \cdot)(\beta, \cdot)}=0
$$

and

$$
\sum_{\alpha, \beta \in R_{+}, r_{\alpha} r_{\beta}=w} k_{\alpha} k_{\beta} B(\alpha, \beta) \Delta_{\alpha} \Delta_{\beta}=0
$$

Proof: Using the definition $\Delta_{\alpha}=\frac{1}{(\alpha, \cdot)}\left(1-r_{\alpha}\right)$ the left hand side of $(2.10)$ can be written as a sum of the following three terms

$$
A=\sum k_{\alpha} k_{\beta} B(\alpha, \beta) \frac{1}{(\alpha, \cdot)(\beta, \cdot)}
$$

$$
\begin{gathered}
B=-\sum k_{\alpha} k_{\beta} B(\alpha, \beta)\left\{\frac{1}{(\alpha, \cdot)\left(r_{\alpha} \beta, \cdot\right)} r_{\alpha}+\frac{1}{(\alpha, \cdot)(\beta, \cdot)} r_{\beta}\right\} \\
C=\sum k_{\alpha} k_{\beta} B(\alpha, \beta) \frac{1}{(\alpha, \cdot)\left(r_{\alpha} \beta, \cdot\right)} r_{\alpha} r_{\beta}
\end{gathered}
$$

with the summations over the same index set as in (2.9) and (2.10).

Let $S=R \cap \operatorname{Im}(w-\mathrm{Id})$ be the normalized root system of the largest dihedral group $W(S)$ containing $w$. If $w=r_{\alpha} r_{\beta}$ then for $\gamma \in S$ we have $r_{\gamma} w r_{\gamma}=w^{-1}$ and hence $r_{r_{\gamma} \alpha} r_{r_{\gamma} \beta}=r_{\beta} r_{\alpha}$. We claim that $r_{\gamma} A=A \forall \gamma \in S$. Indeed we have

$$
\begin{aligned}
r_{\gamma} A & =\sum_{\alpha, \beta \in R_{+}, r_{\alpha} r_{\beta}=w} k_{\alpha} k_{\beta} B(\alpha, \beta) \frac{1}{\left(r_{\gamma} \alpha, \cdot\right)\left(r_{\gamma} \beta, \cdot\right)} \\
& =\sum_{\alpha, \beta \in r_{\gamma} R_{+}, r_{\beta} r_{\alpha}=w} k_{\alpha} k_{\beta} B\left(r_{\gamma} \alpha, r_{\gamma} \beta\right) \frac{1}{(\alpha, \cdot)(\beta, \cdot)} \\
& =\sum_{\alpha, \beta \in r_{\gamma} R_{+}, r_{\beta} r_{\alpha}=w} k_{\alpha} k_{\beta} B(\beta, \alpha) \frac{1}{(\alpha, \cdot)(\beta, \cdot)} \\
& =A
\end{aligned}
$$

since the summation in (2.9) is independent of the choice of $R_{+}$. Let $S_{+}=R_{+} \cap S$ and put $p_{S}=\prod_{\alpha \in S_{+}}(\alpha, \cdot)$. Then $p_{S}$ transforms under the group $W(S)$ according to the sign character and every polynomial in $\mathbb{C}[E]$ transforming under $W(S)$ according to the sign character is divisible in $\mathbb{C}[E]$ by $p_{S}$. Now observe that $p_{S} A \in \mathbb{C}[E]$ transforms 
under $W(S)$ according to the sign character. Hence $A \in \mathbb{C}[E]$. Since $A$ is homogeneous of degree minus two we have $A=0$. This proves (2.9).

Since $w=r_{\alpha} r_{\beta}=r_{r_{\alpha} \beta} r_{\alpha}$ and $B(\alpha, \beta)=B\left(r_{\alpha} \beta, r_{\alpha} \alpha\right)=-B\left(r_{\alpha} \beta, \alpha\right)$ the vanishing of the term (2.12) is clear, and for the term (2.13) we can write $C=-A w=0$. Q.E.D.

Lemma 2.3. For $\xi, \eta \in E$ fixed the bilinear form

$$
B(\lambda, \mu)=(\lambda, \xi)(\mu, \eta)-(\lambda, \eta)(\mu, \xi)
$$

on $E$ satisfies condition (2.8).

Proof: Clearly $B(\mu, \lambda)=-B(\lambda, \mu)$ is an alternating form. For $\lambda \in E, \lambda \neq 0$ we write $\lambda^{\prime}=\sqrt{2}|\lambda|^{-1} \lambda$ and get

$$
B\left(r_{\lambda^{\prime}} \lambda, r_{\lambda^{\prime}} \mu\right)=B\left(-\lambda, \mu-\left(\lambda^{\prime}, \mu\right) \lambda^{\prime}\right)=B(-\lambda, \mu)=B(\mu, \lambda) .
$$

Hence for $\lambda, \mu \in E$ generic we get by continuity

$$
B\left(r_{\nu} \lambda, r_{\nu} \mu\right)=B(\mu, \lambda) \quad \forall \nu \in \operatorname{span}\langle\lambda, \mu\rangle,(\nu, \nu)=2 . \quad \text { Q.E.D. }
$$

The proof of Theorem 1.5 now follows by regrouping the terms in (2.7) as a sum over $\left\{\alpha, \beta \in R_{+} ; r_{\alpha} r_{\beta}=w\right\}$ where $w \in W$ runs over the pure rotations in $W$ and by applying (2.10).

The proof of Theorem 1.6 is just an easy calculation.

$$
\begin{aligned}
& \sum_{j=1}^{n} D_{\xi_{j}}^{2}=\sum_{j=1}^{n}\left(\partial \xi_{j}+\sum_{\alpha \in R_{+}} k_{\alpha}\left(\alpha, \xi_{j}\right) \Delta_{\alpha}\right)^{2} \\
& =\sum_{j=1}^{n}\left\{\partial_{\xi_{j}}^{2}+\sum_{\alpha \in R_{+}} k_{\alpha}\left(\alpha, \xi_{j}\right)\left(\partial_{\xi_{j}} \Delta_{\alpha}+\Delta_{\alpha} \partial_{\xi_{j}}\right)+\sum_{\alpha, \beta \in R_{+}} k_{\alpha} k_{\beta}\left(\alpha, \xi_{j}\right)\left(\beta, \xi_{j}\right) \Delta_{\alpha} \Delta_{\beta}\right\} \\
& =\sum_{j=1}^{n} \partial_{\xi_{j}}^{2}+\sum_{\alpha \in R_{+}} k_{\alpha}\left(\partial_{\alpha} \Delta_{\alpha}+\Delta_{\alpha} \partial_{\alpha}\right)+\sum_{\alpha, \beta \in R_{+}} k_{\alpha} k_{\beta}(\alpha, \beta) \Delta_{\alpha} \Delta_{\beta} .
\end{aligned}
$$

The third term vanishes by Proposition 2.2 and because $\Delta_{\alpha}^{2}=0$. Using Lemma 2.1 we get

$$
\begin{aligned}
\partial_{\alpha} \Delta_{\alpha}+\Delta_{\alpha} \partial_{\alpha} & =\left[\partial_{\alpha}, \Delta_{\alpha}\right]+2 \Delta_{\alpha} \partial_{\alpha} \\
& =\frac{(\alpha, \alpha)}{(\alpha, \cdot)}\left\{r_{\alpha} \partial_{\alpha}-\Delta_{\alpha}\right\}+\frac{2}{(\alpha, \cdot)}\left(1-r_{\alpha}\right) \partial_{\alpha} \\
& =\frac{2}{(\alpha, \cdot)}\left\{\partial_{\alpha}-\Delta_{\alpha}\right\} .
\end{aligned}
$$




\section{$\S 3$. The Opdam shift operators.}

Recall that $D \in \operatorname{End}\left(\mathbb{C}\left[p_{1}, \ldots, p_{m}\right]\right)$ is a differential operator of degree $\leq d$ if and only if

$$
\operatorname{ad}(p)^{d+1}(D)=0 \quad \forall p \in \mathbb{C}\left[p_{1}, \ldots, p_{n}\right] .
$$

Hence the fact that the operators (1.11), (1.16) and (1.17) are differential operators is clear from

$$
\begin{gathered}
\operatorname{ad}(p)\left(D_{\xi}\right)=\operatorname{ad}(p)\left(\partial_{\xi}\right)=-\partial_{\xi}(p) \\
\operatorname{ad}(p)^{2}\left(D_{\xi}\right)=0
\end{gathered}
$$

$\forall p \in \mathbb{C}[E]^{W}, \forall \xi \in E$. Hence Theorem 1.7 is an immediate consequence of Theorem 1.5 and Theorem 1.6.

Theorem 3.1. For the $q$-analogue of the Laplacian we have

$$
\operatorname{Res}\left(p_{S}^{-1} \circ\left\{\sum_{j=1}^{n} D_{\xi_{j}}^{2}(k)\right\} \circ p_{S}\right)=\operatorname{Res}\left(\sum_{j=1}^{n} D_{\xi_{j}}^{2}\left(k+1_{S}\right)\right) .
$$

Proof: First we observe that the left hand side of (3.4) is a well defined endomorphism of $\mathbb{C}[E]^{W}$. We now use Theorem 1.6 and just calculate term by term. For the first term we get

$$
\begin{aligned}
p_{S}^{-1} \circ \sum_{j=1}^{n} \partial_{\xi_{j}}^{2} \circ p_{S} & =\sum_{j=1}^{n} \partial_{\xi_{j}}^{2}+2 \sum_{\alpha \in S_{+}} \frac{1}{(\alpha, \cdot)} \partial_{\alpha}+p_{S}^{-1}\left(\sum_{j=1}^{n} \partial_{\xi_{j}}^{2}\right)\left(p_{S}\right) \\
& =\sum_{j=1}^{n} \partial_{\xi_{j}}^{2}+2 \sum_{\alpha \in S_{+}} \frac{1}{(\alpha, \cdot)} \partial_{\alpha} .
\end{aligned}
$$

For the second term we get

$$
\begin{aligned}
p_{S}^{-1} \circ\left\{2 \sum_{\alpha \in R_{+}} k_{\alpha} \frac{1}{(\alpha, \cdot)} \partial_{\alpha}\right\} \circ p_{S} & =2 \sum_{\alpha \in R_{+}} k_{\alpha} \frac{1}{(\alpha, \cdot)} \partial_{\alpha}+p_{S}^{-1} \cdot\left(2 \sum_{\alpha \in R_{+}} k_{\alpha} \frac{1}{(\alpha, \cdot)} \partial_{\alpha}\right)\left(p_{S}\right) \\
& =2 \sum_{\alpha \in R_{+}} k_{\alpha} \frac{1}{(\alpha, \cdot)} \partial_{\alpha}+2 \sum_{\alpha \in R_{+}, \beta \in S_{+}} k_{\alpha} \frac{(\alpha, \beta)}{(\alpha, \cdot)(\beta, \cdot)} \\
& =2 \sum_{\alpha \in R_{+}} k_{\alpha} \frac{1}{(\alpha, \cdot)} \partial_{\alpha}+2 \sum_{\beta \in S_{+}} k_{\beta} \frac{(\beta, \beta)}{(\beta, \cdot)^{2}} \\
& +2 \sum_{\alpha \in R_{+}, \beta \in S_{+}} k_{\alpha} \frac{(\alpha, \beta)}{(\alpha, \cdot)(\beta, \cdot)} \\
& =2 \sum_{\alpha \in R_{+}} k_{\alpha} \frac{1}{(\alpha, \cdot)} \partial_{\alpha}+2 \sum_{\beta \in S_{+}} k_{\beta} \frac{2}{(\beta, \cdot)^{2}}
\end{aligned}
$$


by the same argument as in the proof of Proposition 2.2.

Finally for the third term we have

$$
\begin{aligned}
p_{S}^{-1} \circ\left\{2 \sum_{\alpha \in R_{+}} k_{\alpha} \frac{1}{(\alpha, \cdot)} \Delta_{\alpha}\right\} \circ p_{S}= & 2 \sum_{\alpha \in R_{+}} k_{\alpha} \frac{1}{(\alpha, \cdot)^{2}}\left\{1-p_{S}^{-1} \circ r_{\alpha} \circ p_{S}\right\} \\
= & 2 \sum_{\alpha \in R_{+}} k_{\alpha} \frac{1}{(\alpha, \cdot)^{2}}\left\{1-\chi_{S}\left(r_{\alpha}\right) r_{\alpha}\right\} \\
= & 2 \sum_{\alpha \in S_{+}} k_{\alpha} \frac{1}{(\alpha, \cdot)^{2}}\left\{1+r_{\alpha}\right\}+2 \sum_{\alpha \in R_{+} \backslash S_{+}} k_{\alpha} \frac{1}{(\alpha, \cdot)} \Delta_{\alpha} \\
= & 2 \sum_{\alpha \in S_{+}} k_{\alpha} \frac{2}{(\alpha, \cdot)^{2}}-2 \sum_{\alpha \in S_{+}} k_{\alpha} \frac{1}{(\alpha, \cdot)} \Delta_{\alpha} \\
& +2 \sum_{\alpha \in R_{+} \backslash S_{+}} k_{\alpha} \frac{1}{(\alpha, \cdot)} \Delta_{\alpha} .
\end{aligned}
$$

Taking all three terms together yields

$$
\begin{aligned}
p_{S}^{-1} \circ\left\{\sum_{j=1}^{n} D_{\xi_{j}}^{2}(k)\right\} \circ p_{S} & =\sum_{j=1}^{n} \partial_{\xi_{j}}^{2}+2 \sum_{\alpha \in R_{+}} k_{\alpha} \frac{1}{(\alpha, \cdot)} \partial_{\alpha}+2 \sum_{\alpha \in S_{+}} \frac{1}{(\alpha, \cdot)} \partial_{\alpha} \\
& +2 \sum_{\alpha \in S_{+}} k_{\alpha} \frac{1}{(\alpha, \cdot)} \Delta_{\alpha}-2 \sum_{\alpha \in R_{+} \backslash S_{+}} k_{\alpha} \frac{1}{(\alpha, \cdot)} \Delta_{\alpha} . \text { Q.E.D. }
\end{aligned}
$$

Corollary 3.2. We have the shift relations

$$
\begin{aligned}
& G\left(1_{S}, k\right) \operatorname{Res}\left(\sum_{j=1}^{n} D_{\xi_{j}}^{2}(k)\right)=\operatorname{Res}\left(\sum_{j=1}^{n} D_{\xi_{j}}^{2}\left(k+1_{S}\right)\right) G\left(1_{S}, k\right) \\
& G\left(-1_{S}, k\right) \operatorname{Res}\left(\sum_{j=1}^{n} D_{\xi_{j}}^{2}(k)\right)=\operatorname{Res}\left(\sum_{j=1}^{n} D_{\xi_{j}}^{2}\left(k-1_{S}\right)\right) G\left(-1_{S}, k\right) .
\end{aligned}
$$

Proof: Indeed we have

$$
\begin{aligned}
\operatorname{Res}\left(p_{S}^{-1} D_{\pi_{S}}(k)\right) \operatorname{Res}\left(\sum_{j=1}^{n} D_{\xi_{j}}^{2}(k)\right) & =\operatorname{Res}\left(\sum_{j=1}^{n} p_{S}^{-1} D_{\pi_{S}}(k) D_{\xi_{j}}^{2}(k)\right) \\
& =\operatorname{Res}\left(\sum_{j=1}^{n} p_{S}^{-1} D_{\xi_{j}}^{2}(k) D_{\pi_{S}}(k)\right) \\
& =\operatorname{Res}\left(\sum_{j=1}^{n} p_{S}^{-1} D_{\xi_{j}}^{2}(k) p_{S}\right) \operatorname{Res}\left(p_{S}^{-1} D_{\pi_{S}}(k)\right) \\
& =\operatorname{Res}\left(\sum_{j=1}^{n} D_{\xi_{j}}^{2}\left(k+1_{S}\right)\right) \operatorname{Res}\left(p_{S}^{-1} D_{\pi_{S}}(k)\right)
\end{aligned}
$$


which proves (3.5). The relation (3.6) is proved similarly.

Q.E.D.

Theorem 3.3. As endomorphisms of $\mathbb{C}[E]$ the operators

$$
\begin{aligned}
& E=\frac{1}{2} \sum_{j=1}^{n}\left(\xi_{j, .}\right)^{2} \\
& H=\sum_{j=1}^{n}\left(\xi_{j,}\right) \partial_{\xi_{j}}+\left(\frac{n}{2}+\sum_{\alpha \in R_{+}} k_{\alpha}\right) \\
& F=-\frac{1}{2} \sum_{j=1}^{n} D_{\xi_{j}}^{2}
\end{aligned}
$$

satisfy the commutation relations of $\operatorname{sl}(2)$ :

$$
[H, E]=2 E,[H, F]=-2 F,[E, F]=H .
$$

Proof: The Euler operator $\sum_{j=1}^{n}\left(\xi_{j,}.\right) \partial_{\xi_{j}}$ acts as multiplication by $d$ on the space of homogeneous polynomials in $\mathbb{C}[E]$ of degree $d$. Hence the commutation relations $[H, E]=2 E,[H, F]=-2 F$ rephrase that $E$ and $F$ are homogeneous of degree plus and minus two respectively.

Since $\left[p, \Delta_{\alpha}\right]=0 \forall p \in \mathbb{C}[E]^{W}, \forall \alpha \in R$ we get

$$
\left[E, D_{\xi}\right]=\left[E, \partial_{\xi}\right]=-(\xi, \cdot) \quad \forall \xi \in E,
$$

and therefore

$$
\begin{aligned}
{[E, F] } & =-\frac{1}{2} \sum_{j=1}^{n}\left[E, D_{\xi_{j}}^{2}\right] \\
& =\frac{1}{2} \sum_{j=1}^{n}\left\{\left(\xi_{j, .}\right) D_{\xi_{j}}+D_{\xi_{j}}\left(\xi_{j, .}\right)\right\} \\
& =\sum_{j=1}^{n}\left(\xi_{j, .}\right) D_{\xi_{j}}+\frac{1}{2} \sum_{j=1}^{n}\left[D_{\xi_{j}},\left(\xi_{j, .}\right)\right] \\
& =\sum_{j=1}^{n}\left(\xi_{j, .}\right) D_{\xi_{j}}+\frac{n}{2}+\frac{1}{2} \sum_{j=1}^{n} \sum_{\alpha \in R_{+}} k_{\alpha}\left(\alpha, \xi_{j}\right)\left[\Delta_{\alpha},\left(\xi_{j, .}\right)\right] \\
& =\sum_{j=1}^{n}\left(\xi_{j, .}\right) \partial_{\xi_{j}}+\sum_{\alpha \in R_{+}} k_{\alpha}(\alpha, \cdot) \Delta_{\alpha}+\frac{n}{2}+\sum_{\alpha \in R_{+}} k_{\alpha} r_{\alpha} \\
& =\sum_{j=1}^{n}\left(\xi_{j, .}\right) \partial_{\xi_{j}}+\left(\frac{n}{2}+\sum_{\alpha \in R_{+}} k_{\alpha}\right) .
\end{aligned}
$$


Here we have used that for $\xi \in E$

$$
\begin{aligned}
{\left[\Delta_{\alpha},(\xi, \cdot)\right] } & =-\frac{1}{(\alpha, \cdot)}\left[r_{\alpha},(\xi, \cdot)\right] \\
& =-\frac{1}{(\alpha, \cdot)}\left\{\left(r_{\alpha} \xi, \cdot\right)-(\xi, \cdot)\right\} r_{\alpha} \\
& =(\alpha, \xi) r_{\alpha} .
\end{aligned}
$$

Proposition 3.4. Using the inner product $(\cdot, \cdot)$ on $E$ we have an isomorphism between $\mathbb{C}[E]$ and $\mathbb{C}\left[E^{*}\right]$. For $p \in \mathbb{C}[E]$ we write $\pi \in \mathbb{C}\left[E^{*}\right]$ for the corresponding element. For $p \in \mathbb{C}[E]$ homogeneous of degree $d$ we have

$$
D_{\pi}=(-1)^{d} \frac{1}{d !} \operatorname{ad}(F)^{d}(p) .
$$

Proof: Clearly $\operatorname{ad}(H) D_{\pi}=-d D_{\pi}$ and by Theorem 1.5 we have $\operatorname{ad}(F) D_{\pi}=0$. Using (3.11) and induction on $d$ (assuming $\pi$ to be a monomial as in (1.9) with $\left.d=d_{1}+\cdots+d_{n}\right)$ it is easy to see that

$$
(-1)^{d} \frac{1}{d !} \operatorname{ad}(E)^{d}\left(D_{\pi}\right)=p
$$

and hence

$$
\operatorname{ad}(E)^{d+1}\left(D_{\pi}\right)=0 \text {. }
$$

By standard representation theory of $s l(2)$ we conclude (3.12).

Q.E.D.

Corollary 3.5. For $\pi \in \mathbb{C}\left[E^{*}\right]^{W}$ we have

$$
\operatorname{Res}\left(p_{S}^{-1} \circ D_{\pi}(k) \circ p_{S}\right)=\operatorname{Res}\left(D_{\pi}\left(k+1_{s}\right)\right) .
$$

Proof: This is easily derived from Theorem 3.1 and Proposition 3.4.

Q.E.D.

The proof of Theorem 1.9 now goes along the same lines as the proof of Corollary 3.2.

Remark 3.6. The above type of arguments to use an $s l(2)$ to reduce the computation of higher order operators to those of the second order one go back to Harish-Chandra $[\mathrm{Ha}]$.

Acknowledgements: The author would like to thank the organizers of this conference for the invitation, and in particular Bill Barker for the hospitality at Bowdoin College. 


\section{References}

[BGG] I.N. Bernstein, I.M. Gel'fand, S.I. Gel'fand, Schubert cells and the cohomology of G/P, Russ. Math. Surveys 28 (1973), 1-26.

[De 1] M. Demazure, Désingularisation des variétés de Schubert généralisés, Ann. Sc. Éc. Norm. Sup. 7 (1974), 53-88.

[De 2] M. Demazure, Une nouvelle formule des caractères, Bull. Sc. Math. 98 (1974), 163-172.

[Du] C.F. Dunkl, Differential-difference operators associated to reflection groups, Trans. AMS Vol. 311 (1989), 167-183.

[Ha] Harish-Chandra, Differential operators on a semisimple Lie algebra, Amer. J. Math. 79 (1957), 87-120, or the Collected Works, Vol.2, 243-276.

[HO] G.J. Heckman, E.M. Opdam, Root systems and hypergeometric functions I, Comp. Math. 64 (1987), 329-352.

[He 1] G.J. Heckman, Root systems and hypergeometric functions II, Comp. Math. 64 (1987), 353-373.

[He 2] G.J. Heckman, Hecke algebras and hypergeometric functions, Inv. Math. (to appear).

[Ma] I.G. Macdonald, Some conjectures for root systems, Siam J. Math. Analysis, Vol. 13, No. 6 (1982), 988-1007.

[Mo] J. Moser, Three integrable systems connected with isospectral deformation, Adv. Math. 16 (1975), 197-220.

[OP] M.A. Olshanetsky and A.M. Perelomov. Completely integrable systems connected with semisimple Lie algebras, Inv. Math. 37 (1976), 93-108.

[Op 1,2] E.M. Opdam, Root systems and hypergeometric functions III, IV, Comp. Math. 67 (1988), 21-49 and 191-209.

[Op 3] E.M. Opdam, Some applications of hypergeometric shift operators, Inv. Math. (to appear).

[Op 4] E.M. Opdam, Normalization problems for hypergeometric functions associated to root systems (in preparation).

[YS] T. Yano, J. Sekiguchi, The microlocal structure of weighted homogeneous polynomials associated with Coxeter systems I, II, Tokyo J. Math. Vol. 2, No. 2 (1979), 193-219 and Vol. 4, No. 1 (1981), 1-34. 
University of Nijmegen, Mathematisch Instituut, Toernooiveld, 6525 ED Nijmegen. 